\title{
Relación grupos estratégicos-desempeño empresarial: análisis empírico de la industria farmacéutica española
}

\author{
Piña Mavárez, Enzo Enrique* \\ Suárez Serrano, Eugenia**
}

\section{Resumen}

El objetivo de este trabajo es analizar la relación entre el concepto de grupo estratégico y el desempeño empresarial en la industria farmacéutica española. Para establecer los grupos estratégicos hemos seleccionado una muestra de 45 laboratorios y hemos utilizado variables propias del sector para el periodo 2000-2003, a las que hemos aplicado un análisis cluster no jerárquico para clasificar las empresas en tres grupos y posteriormente la Prueba de Kruskal-Wallis para evaluar la relación entre los grupos y las variables de desempeño, considerando además el riesgo asumido por las empresas. Los resultados nos revelan que no existe relación entre las estrategias de los grupos y su desempeño económico. No obstante, se han encontrado diferencias en dos de los tres indicadores ajustados por el riesgo para uno de los tres grupos. Finalmente, podemos concluir que en la industria farmacéutica española las diferentes empresas han encontrado alternativas estratégicas, algunas de ellas, relacionadas con innovaciones de carácter incremental para alcanzar resultados similares al de sus competidores, además de comprobarse la importancia del factor riesgo dentro de esta industria.

Palabras clave: Grupos estratégicos, desempeño empresarial, industria farmacéutica.

Recibido: 11-02-08. Aceptado: 26-05-09

* Profesor Asociado de la Facultad de Farmacia y Bioanálisis de la Universidad de Los Andes. Doctor en Dirección de Empresas por la Universidad de Oviedo, España. Para correspondencia: correo: epmavarez@ula.ve.

** Profesora Titular de Organización de Empresas de la Facultad de Ciencias Económicas y Empresariales de la Universidad de Oviedo, España. Doctora en Dirección de Empresas por la Universidad de Oviedo. Correo: meugenia@uniovi.es. 


\title{
The Relationship of Strategic Groups to Business Performance: Empirical Analysis of the Spanish Pharmaceutical Industry
}

\begin{abstract}
The objective of this paper is to analyse the relationship between the concept of strategic groups and corporate performance of the Spanish pharmaceutical industry. In order to establish the strategic groups, a sample from 45 different laboratories was selected and variables from the sector were used for the period 2000-2003, to which a non-hierarchical cluster analysis was applied to classify the industries in three groups. Afterwards, the Kruskal-Wallis test was used for evaluating relationships among the groups and their performance variables, considering furthermore, the risk assumed by the companies. Results revealed that there is no connection between the strategies used by the groups and their economic performance. However, differences have been found in two out of three indicators adjusted for risk for one of the three groups. Finally, conclusions are that in the Spanish pharmaceutical industry, different firms have found strategic alternatives, some of them related to innovations of an incremental character, for reaching results similar to those of their competitors; conclusions also prove the importance of the risk factor within this industry.
\end{abstract}

Key words: Strategic groups, business performance, pharmaceutical industry.

\section{Introducción}

La teoría de grupos estratégicos ha sido empleada en numerosos trabajos para explicar las diferencias entre empresas, tanto si nos referimos a las acciones estratégicas emprendidas por éstas, como para explicar los diferentes niveles de rentabilidad obtenidos. En tal sentido, existen varias líneas de investigación que están guiando dichos trabajos hacia varios campos de la dirección estratégica. Así, mientras que la economía industrial, identifica a la estructura de la industria como el principal determinante de sus resultados empresariales (Bain, 1956), la perspectiva de recursos y capacidades (Barney, 1991; Peteraf, 1993) traslada el análisis hacia el interior de la empresa e interpreta que los recursos propios de la misma van a ser más influyentes.
Entre la industria y la empresa se interpone el concepto de grupo estratégico como una herramienta de análisis para estudiar la competencia en los sectores industriales, con una visión más completa e integrada que la que ofrece el análisis de una sola empresa o la que permite el estudio agregado de los sectores.

Ahora bien, dependiendo del sector industrial en cuestión, los resultados obtenidos por las empresas podrán ser más o menos homogéneos, dependiendo de los distintos niveles de riesgo asumidos por las mismas. Por lo tanto, en la práctica podrían existir dentro de una misma industria diversas empresas que asuman niveles de riesgo distintos y que con seguridad influyen en los resultados empresariales obtenidos al enfrentar distintos niveles de incertidumbre.

La industria farmacéutica española es catalogada como una industria alta- 
mente innovadora dentro del conjunto total de la industria. Ahora bien, este proceso innovador resulta costoso y de mucho riesgo debido a la incertidumbre que rodea a las actividades de Investigación y Desarrollo (I+D) farmacéutica, la cual hace que la gran mayoría de los compuestos descubiertos con actividad medicinal no se conviertan en medicamentos patentados al no poder pasar los estrictos ensayos clínicos que se le aplican a estos productos. Por otro lado, la empresa poco innovadora también asume elevados niveles de riesgo, precisamente por no contar con recursos óptimos para competir con las grandes multinacionales que tienen asegurado un flujo constante de nuevos lanzamientos de productos al mercado y tener que enfrentar en situación de desventaja competitiva a estas poderosas compañías.

Con este escenario de por medio, este trabajo pretende analizar la relación entre grupos estratégicos y desempeño empresarial en la industria farmacéutica española, sopesando, por supuesto, los niveles de riesgo asumidos por los laboratorios farmacéuticos establecidos en territorio español durante el período 2000-2003, coincidiendo casi en su totalidad con el pacto de estabilidad Gobierno-industria farmacéutica 2001-2004.

En cuanto a los aspectos metodológicos, se elaboró una encuesta con la ayuda de los industriales y se enviaron 125 encuestas postales, obteniendo una tasa de respuesta de $36 \%$. En tal sentido, la muestra quedó conformada por $45 \mathrm{em}$ presas, de las cuales 22 son de capital nacional, 18 de capital extranjero y 5 de capital mixto. Las 45 empresas de la muestra representan un $68 \%$ de la pro- ducción total de la industria farmacéutica para el año 2003.

Hemos de señalar, que para establecer los grupos estratégicos seguimos el criterio expuesto por Cool y Schendel (1987), el cual separa las variables estratégicas en dos grupos: las que describan el alcance de las actividades y las que representan el compromiso de recursos. En resumen, las dimensiones estratégicas que definen los principales comportamientos en la industria en cuestión se reúnen en siete dimensiones que enfatizan sobre la conducta innovadora por considerar esta actividad prioritaria dentro del sector.

Para analizar una posible relación entre estrategia de grupos y resultados, atenderemos a las recomendaciones ofrecidas por algunos autores como McGee et al. (1995), quienes sugieren la utilización de más de un indicador del resultado empresarial. Este criterio es acorde con las consideraciones de Cool y Schendel (1987) cuando afirman que el resultado es un concepto de naturaleza multidimensional. Por ello tendremos en cuenta como indicadores de resultados al Margen de Beneficios, a la Rentabilidad Económica y a la Rentabilidad Financiera. Finalmente para comprobar si existe o no relación entre los grupos y los indicadores de resultados emplearemos la Prueba de KruskalWallis para realizar este contraste.

\section{Soporte teórico de la investigación y planteamiento de hipótesis}

El término grupos estratégicos fue introducido por Hunt (1972) y su uso fue extendiéndose con rapidez. Porter (1979) definió los grupos estratégicos como un 
conjunto de empresas en un sector industrial que desarrollan comportamientos similares a lo largo de una serie de variables de decisión clave y es precisamente la elección de estas variables una de las mayores dificultades para aplicar el concepto de grupos estratégicos a un sector industrial al no existir un procedimiento óptimo universalmente aceptado.

Al respecto Cool y Schendel (1987) establecen diferencias entre dos tipos de actividades, las relacionadas con el "alcance de las actividades" y las concernientes al "compromiso de recursos" a partir de las cuales las empresas determinan su estrategia competitiva. El alcance de las actividades estaría representado por ciertas decisiones relacionadas con los productos y/o servicios que se ofrecen y los segmentos de mercado, así como el alcance geográfico de la estrategia. Los compromisos de recursos son definidos como el despliegue de recursos que se contienen en la estrategia de negocio -financieros, humanos, materiales....- $(\mathrm{Pe}-$ layo y Vargas, 2004).

Por otro lado, la posibilidad de que los grupos establecidos en una industria influyan en los resultados de las organizaciones es una de las cuestiones más importantes que plantea la perspectiva de grupos estratégicos (Cool y Schendel, 1988). En tal sentido, de existir diferencias significativas en los resultados entre las distintas agrupaciones de empresas, significaría que las estrategias empleadas por las empresas serían responsables de dichas diferencias, lo que llevaría a los integrantes de una industria a identificar las estrategias más deseables, es decir, aquellas que ofrezcan ventajas competitivas sostenibles.
Sin embargo, algunos investigadores revelan la dificultad existente para adoptar una estrategia similar a la de un grupo estratégico distinto, debido a la existencia de algunos condicionantes o "recursos colectivos" que protegen la posición de los miembros del grupo y plantean serios inconvenientes a los posibles entrantes. Esta figura se denomina barreras a la movilidad y es un concepto muy extendido dentro de la literatura de grupos estratégicos ya que apoya el supuesto de existencia de diferencias de resultados entre grupos. Ahora bien, los resultados empíricos relacionados con este planteamiento son contradictorios. Al respecto, algunos investigadores, entre ellos Lewis y Thomas (1990), Cool y Dierickx (1993) y Reger y Huff (1993), comprobaron empíricamente la existencia de grupos estratégicos, pero admitiendo que dentro de esas empresas que adoptaban estrategias similares existía cierta heterogeneidad y que no existía relación significativa clara entre las estrategias y las rentabilidades de los grupos.

Este tipo de evidencia podría explicarse atendiendo a las competencias distintivas propias de cada empresa lo que les hace implantar una estrategia diferenciada incluso de los mismos miembros de su grupo (Chi, 1994). Los mecanismos de aislamiento expresan que las estrategias seguidas por los miembros de un grupo, aunque similares, pueden ser apoyadas por activos muy específicos desarrollados en el seno de cada empresa, lo que les permite implantar su estrategia de forma individual y obtener resultados diferenciados.

Por lo tanto, en este trabajo enfatizaremos en cuanto a la contrastación em- 
pírica de la relación grupos estratégicosdesempeño empresarial en la industria farmacéutica española, utilizando como medidas de desempeño tres indicadores muy utilizados dentro de las investigaciones de grupos estratégicos, ellos son el margen de beneficios, la rentabilidad económica y la rentabilidad financiera, por lo que nuestra primera hipótesis a plantear queda redactada de la siguiente forma:

Hipótesis 1: Los grupos estratégicos establecidos en la industria farmacéutica española presentarán diferencias de desempeño en términos de los siguientes resultados:

a) Margen de beneficios

b) Rentabilidad económica

c) Rentabilidad financiera

Como señalan Flavian y Polo (2000) y Claver et al. (2002), en la mayor parte de los estudios previos la atención se ha centrado fundamentalmente en demostrar la existencia de diferencias de resultados entre grupos estratégicos sin considerar los niveles de riesgo asumido. Siguiendo a los autores antes mencionados, en este trabajo se ha medido el riesgo soportado por las empresas utilizando la desviación estándar de las correspondientes medidas de resultados para cada empresa como un indicativo del nivel de incertidumbre que rodea a su desempeño.

Hemos señalado que el proceso innovador en la industria farmacéutica española -basado en la I+D de nuevas entidades químicas- resulta altamente incierto y arriesgado, no sólo por la erosión competitiva que supone la amenaza de productos sustitutivos, sino también a las dificultades inherentes al propio proceso innovador. Por tal motivo hemos conside- rado sopesar los riesgos asumidos por los laboratorios farmacéuticos y compararlos con las agrupaciones obtenidas. Nuestra hipótesis en este sentido queda establecida de la siguiente forma:

Hipótesis 2: Los grupos estratégicos establecidos en la industria farmacéutica presentarán diferencias en cuanto a los niveles de riesgo asumido reflejados en:

a) Margen de beneficios

b) Rentabilidad económica

c) Rentabilidad financiera

Finalmente, hemos de considerar las dimensiones de resultados ajustados por el riesgo asumido por cada empresa. Si bien es cierto que dentro de la industria farmacéutica algunas estrategias -como la adopción de una I+D radical- podría generar un nivel de resultados mayor, no es menos cierto que dichas empresas también pueden verse afectadas por un mayor nivel de riesgo asumido, de tal forma que, el riesgo sería quizás uno de los principales factores que condicione la obtención de resultados en esta industria. Basados en esta premisa formulamos la siguiente hipótesis:

Hipótesis 3: El nivel de resultados ajustado por el riesgo soportado por las empresas que pertenecen a grupos estratégicos distintos presentarán diferencias en cuanto.

a) Margen de beneficios

b) Rentabilidad económica

c) Rentabilidad financiera

\section{Obtención de los grupos estratégicos}

Hemos de señalar, que para establecer los grupos estratégicos seguimos 
el criterio expuesto por Cool y Schendel (1987), por lo que las dimensiones estratégicas se reúnen en siete variables que representan los principales comportamientos estratégicos de las empresas del sector, enfatizando, por supuesto, sobre la conducta innovadora por considerar esta actividad la piedra angular en cuanto a los mecanismos competitivos de esta industria (Cuadro 1).

Las unidades para medir los siete aspectos estratégicos planteados son muy disímiles (euros, países, segmentos de la terapéutica, personas, número de patentes), por lo que procedimos a aplicar un procedimiento de tipificación convirtiendo a cada variable a unas puntuaciones estándar restando la media y dividiendo entre la desviación típica de cada variable siguiendo uno de los procedimientos recomendados para tal fin (Pérez, 1997; Hair, Anderson, Tatham y Black, 1999).

Para la obtención de los grupos estratégicos hemos aplicado un análisis cluster no jerárquico de la K-medias y se han obtenido tres conglomerados homo- géneos utilizando la distancia euclídea respecto al centroide de cada grupo. Es preciso señalar que el número de grupos fue establecido en tres tomando en cuenta la opinión de los industriales que además concuerda con el análisis expuesto por Solà (2000) al concluir luego de un profundo estudio teórico de la industria farmacéutica española que podría ser ese el número de conjuntos con características similares establecidos en esta industria.

Con respecto a este punto, Dranove et al. (1998) recomiendan que los investigadores identifiquen los G.E. a priori basados en un profundo conocimiento de la industria, debido a que este procedimiento ayuda a que las agrupaciones obtenidas por el método estadístico empleado, estén más adecuadas a la realidad al definir previamente algunas barreras y límites de los grupos en un período temporal. El resultado del análisis cluster realizado se muestra a continuación, junto al número de empresas por grupo (Tabla 1).

Las puntuaciones que aparecen para cada variable en la Tabla 1 repre-

\section{Cuadro 1 \\ Aspectos estratégicos e indicadores utilizados}

\begin{tabular}{ll}
\hline \multicolumn{1}{c}{ Variables (alcance de las actividades) } & \multicolumn{1}{c}{ Indicadores } \\
\hline Internacionalización (INTERNAC) & Número de países en los que opera \\
Diversificación (DIVERS) & Número de segmentos terapéuticos atendidos \\
Eficiencia de la I+D (PATENTE) & Número de patentes concedidas \\
\hline \multicolumn{1}{c}{ Variables (compromiso de recursos) } & \multicolumn{1}{c}{ Indicadores } \\
\hline Tamaño (EMPLEO) & Número total de empleados \\
Estrategia de I+D (INVES) $\quad$ (EMPLID) & $\begin{array}{l}\text { Gastos de I+D } \\
N^{\circ} \text { empleados dedicados a la I+D en España }\end{array}$ \\
Economías de escala (VENTAS) & Ventas \\
\hline
\end{tabular}

Fuente: Elaboración propia. 
sentan los centros de los conglomerados para cada grupo, alrededor de los cuales se agregan cada una de las puntuaciones de cada variable cercanas a dichos centros. Los tres grupos obtenidos del análisis cluster nos muestran que el $80 \%$ de las empresas de la muestra se ubican en el grupo número 3 , quedando el grupo número 1 compuesto por 3 empresas ( $7 \%$ y el grupo 2 por 6 compañías (13\%).

La Tabla 2 presenta el análisis de la varianza, el cual nos indica que todas las variables son muy explicativas (Prob. menor a 0.05), es decir, todas presentan diferencias significativas entre los distintos clusters.

A continuación se ofrece la descripción de los grupos.

El grupo estratégico 1 (G.E.1) es el grupo menos numeroso al estar constituido por tres empresas, todas ellas de capital nacional y de carácter multinacional. Son laboratorios bastante grandes y con una arraigada tradición en España.

\section{Tabla 1}

\section{Centros de los clusters y número de empresas}

\begin{tabular}{lccc}
\hline Variables & Cluster 1 & Cluster 2 & Cluster 3 \\
\hline Ventas & 1.783 & 1.681 & -.428 \\
Empleo & 2.571 & 1.061 & -.391 \\
CATERAP & 1.651 & .991 & -.302 \\
EMPLID & 3.334 & .296 & -.327 \\
INVDES & 2.294 & 1.321 & -.411 \\
TOTALPAT & -.037 & 1.850 & -.305 \\
INTERNAC & .588 & 1.672 & -.327 \\
No empresas & 3 & 6 & 36 \\
\hline
\end{tabular}

Fuente: Elaboración propia.

Tabla 2

Análisis de la varianza (ANOVA)

\begin{tabular}{lcccccc}
\hline Variables & $\begin{array}{c}\text { Media suma } \\
\text { cuad. grupos }\end{array}$ & gl & $\begin{array}{c}\text { Media suma } \\
\text { cuad. errores }\end{array}$ & gl & $\mathrm{F}$ & Prob. \\
\hline Ventas & 16.564 & 2 & .259 & 42 & 63,988 & 0.00 \\
Empleo & 16.058 & 2 & .283 & 42 & 56,751 & 0.00 \\
CATERAP & 8.692 & 2 & .634 & 42 & 13,716 & 0.00 \\
EMPLID & 18.870 & 2 & .149 & 42 & 126,598 & 0.00 \\
INVDES & 16.191 & 2 & .277 & 42 & 58,529 & 0.00 \\
TOTALPAT & 11.955 & 2 & .478 & 42 & 24,995 & 0.00 \\
INTERNAC & 10.849 & 2 & .531 & 42 & 20,431 & 0.00 \\
\hline
\end{tabular}

Fuente: Elaboración propia. 
La media del número de empleados es de 1572 y además es el grupo que dedica mayor personal a actividades de I+D. Consecuentemente es el grupo que más invierte en ese proceso y el que mayor cantidad de segmentos terapéuticos abarca, es decir, se encuentra más diversificado. No obstante, ese esfuerzo en I+D no genera un elevado número de patentes de medicamentos. Además la presencia internacional de las empresas que conforman el grupo tampoco es la más elevada. A este grupo lo hemos denominado "Multinacionales españolas".

EI grupo estratégico 2 (G.E.2) lo constituyen 6 empresas multinacionales de capital extranjero; al igual que el G.E.1 son empresas bastante grandes tanto en número de empleados como en volumen de ventas (aunque en promedio un poco menor que el G.E.1). A pesar de poseer un número intermedio de personas dedicadas a la I+D en el país, y dedicar una cantidad de recursos económicos moderada a esta actividad, es el grupo que mayor número de patentes sobre medicamentos de uso humano se les concedió, con un promedio de 84 patentes farmacéuticas concedidas durante el periodo por cada empresa. Se encuentran bastante diversificados hacia varias categorías de la terapéutica y es el grupo más internacionalizado con presencia en un promedio de 130 países, lo que brinda una idea de su imagen y poder de negociación. A este grupo lo denominamos "Multinacionales extranjeras".

El grupo estratégico 3 (G.E.3) lo conforman 36 empresas, la mayoría empresas pequeñas y medianas de diverso capital (nacional, extranjero y mixto). Su esfuerzo innovador resulta ser mucho menor que el G.E.1 y G.E.2 al ser el grupo que menos personal y recursos dedica a la I+D, por lo cual, es también el grupo que menor cantidad de patentes farmacéuticas generó durante el periodo de estudio. Están presentes en menos países que el G.E.1 (casi un 50\% menos) y se encuentran especializadas en pocas áreas de la terapéutica. En consecuencia, podemos denominar a este grupo "Empresas especializadas".

\section{Resultados y Discusión}

\subsection{Diferencias de resultados económicos}

Como indicamos en los apartados anteriores, son tres los indicadores de resultados económicos que nos permitirán contrastar las hipótesis señaladas, ellos son: el margen de beneficios (MARBEN), la rentabilidad económica (RENTECO) y la rentabilidad financiera (RENTFIN). A continuación contrastaremos nuestra hipótesis número 1 :

H1: Los grupos estratégicos establecidos en la industria farmacéutica presentarán diferencias de desempeño en términos de los siguientes resultados:

a) Margen de beneficios

b) Rentabilidad económica

c) Rentabilidad financiera

La Tabla 3 muestra los valores que toma la media aritmética simple de las diferentes medidas de resultados para cada uno de los grupos, y para el promedio de la industria durante el periodo de estudio. Aquí podemos notar por una simple comparación que no existe una gran diferencia entre los promedios de resultados obtenidos por cada uno de los gru- 


\section{Tabla 3}

Media de los resultados económicos para los grupos

\begin{tabular}{cccc}
\hline Grupos & MARBEN & RENTECO & RENTFIN \\
\hline G.E.1 & 9.43 & 8.72 & 15.36 \\
G.E.2 & 5.33 & 8.10 & 15.56 \\
G.E.3 & 5.52 & 6.89 & 14.36 \\
Pr. Ind. & 5.76 & 7.17 & 14.58 \\
\hline
\end{tabular}

Fuente: Elaboración propia.

pos, sin embargo, aplicaremos más adelante un test estadístico para corroborar esta sospecha.

El procedimiento a seguir en cada uno de los contrastes será el propuesto por Fiegenbaum y Thomas (1990). Por lo tanto, para las dimensiones del resultado económico tomaremos como referencia la ecuación que refleja la igualdad del resultado medio obtenido por las empresas de cada grupo en el periodo estudiado. Si la probabilidad asociada a la igualdad de medias es especialmente baja podremos rechazar su validez, y por tanto, admitir que los resultados obtenidos por cada grupo presentan diferencias significativas. La ecuación que nos servirá de referencia será:

$$
\overline{\mathrm{X}} \mathrm{ri1}=\ldots \overline{\mathrm{X}} \mathrm{rj2}=\ldots \overline{\mathrm{X}} \mathrm{rk} 3
$$

donde i, j y k representan las empresas que forman los grupos 1, 2 y 3 respectivamente y $\overline{\mathrm{X}}$ r representa la media de cada uno de los indicadores de resultados obtenidos por las empresas del grupo.

En primer lugar, contrastaremos las hipótesis con relación a las diferentes dimensiones del resultado económico, para lo cual aplicaremos la prueba de Kruskal-Wallis considerando las dimensiones de resultados individualmente. Este test constituye un equivalente no paramétrico del ANOVA de un factor, y es útil para comparaciones de $k$ poblaciones si los supuestos de normalidad y homocedasticidad requeridos para la realización de un ANOVA son rechazados.

En el caso que nos ocupa, no existe la certeza de que los supuestos de normalidad y homocedasticidad se cumplan al $100 \%$ en las distintas agrupaciones, tras aplicar los test de Shapiro-Wilk ${ }^{1}$ con cada uno de los indicadores para la normalidad y la prueba M. de Box en el caso de la homocedasticidad.

A continuación se adjunta la Tabla 4 con los resultados correspondientes a la Prueba de Kruskal-Wallis que mide la

1 Existen varias pruebas para la constatación del supuesto de normalidad, entre ellas optamos por la prueba de Shapiro-Wilk por ser la recomendada en caso de poblaciones pequeñas. En caso de poblaciones grandes una de las más utilizadas es la prueba de Kolmogorov-Smirnov. 


\section{Tabla 4}

Prueba de Kruskal-Wallis para las dimensiones económicas

\begin{tabular}{cccc}
\hline & MARBEN & RENTECO & RENTFIN \\
\hline Chi-cuadrado & 3.167 & .542 & .227 \\
gl & 2 & 2 & 2 \\
Sig. asintótica & 0.205 & 0.763 & 0.893 \\
\hline
\end{tabular}

Fuente: Elaboración propia.

posible relación entre los grupos y los resultados, medidos por las tres dimensiones de desempeño consideradas individualmente, lo que nos permitirá contrastar la hipótesis planteada al atender la significación de la prueba -de obtener una significación menor a 0.05 contrastaremos positivamente nuestra hipótesis; de lo contrario nuestra primera hipótesis quedará rechazada-.

Los resultados que aparecen en la Tabla 4 nos muestran que si centramos la atención en cada uno de los indicadores económicos considerados de forma individual nos lleva a concluir que no se aprecian diferencias en los resultados en relación al margen de beneficios, a la rentabilidad económica y a la rentabilidad financiera (Prob. mayores a 0.05), por tanto, no podemos concluir que existan diferencias significativas de resultados entre los grupos.

\subsection{Diferencias de riesgo entre grupos}

Como explicamos anteriormente, las empresas asumen distintos niveles de riesgo al momento de establecer su estrategia. La hipótesis referida a este apartado es la siguiente:

H2: Los grupos estratégicos establecidos en la industria farmacéutica pre- sentarán diferencias en cuanto a los niveles de riesgo asumido reflejados en:

a) Margen de beneficios

b) Rentabilidad económica

c) Rentabilidad financiera

En base a esto, mediremos el riesgo soportado por las empresas siguiendo el procedimiento empleado por Cool y Schendel (1987), calculando la desviación estándar de cada uno de los indicadores de resultados obtenidos por dicha empresa durante los años que integran el periodo. La ecuación que tomaremos como referencia para realizar el contraste será:

$$
\sigma r i 1=\ldots \sigma r j 2=\ldots \text { ork } 3
$$

donde $\mathrm{i}, \mathrm{j}$ y $\mathrm{k}$ representan las empresas que forman los grupos 1, 2 y 3 respectivamente y $\sigma$ r representa la desviación estándar de los resultados obtenidos por las empresas del grupo.

En la Tabla 5 vemos los valores medios de las desviaciones estándar de cada uno de los resultados para las empresas de cada grupo y del promedio industrial.

Las dispersiones obtenidas para cada uno de los resultados se encuentran bastante cerca, sobre todo en el caso de los indicadores margen de beneficios y rentabilidad económica, mostrándose 
una mayor separación con respecto al indicador rentabilidad financiera, en donde las empresas del G.E.1 obtienen una menor dispersión que sus rivales. Seguidamente, en la Tabla 6 se muestra la prueba de Kruskal-Wallis para las desviaciones de los resultados de forma individual, lo cual nos permitirá llevar a cabo el contraste.

Como revela la tabla anterior, la prueba de Kruskal-Wallis no nos confirma que estadísticamente exista diferencias significativas (Sig. mayor a 0.05 en dos de los tres indicadores). Es decir, en relación a los diferentes niveles de riesgo soportado por los distintos grupos estratégicos, hemos constatado que no existen indicios para afirmar que consten diferencias significativas entre grupos en cuanto a la dispersión obtenida referida al margen de beneficios y la rentabilidad económica, mientras que apenas se observan diferencias significativas en el riesgo so- portado por las empresas de los diferentes grupos con respecto a la rentabilidad financiera, lo que nos indica que el nivel de riesgo soportado por las empresas que pertenecen a distintos grupos estratégicos es similar, o al menos, no es muy diferente, en dos de los tres indicadores utilizados.

\subsection{Diferencias de resultados ajustados por el riesgo soportado}

A continuación, hemos de contrastar la hipótesis referida a los resultados ajustados por el riesgo que asumen las empresas:

H3: El nivel de resultados ajustado por el riesgo soportado por las empresas que pertenecen a G.E. distintos presenta diferencias en cuanto a:
a) Margen de beneficios
b) Rentabilidad económica
c) Rentabilidad financiera

Tabla 5

Desviaciones estándar de los resultados para cada grupo

\begin{tabular}{cccc}
\hline Grupos & oMARBEN & oRENTECO & $\sigma$ RENTFIN \\
\hline G.E.1 & 1.5457 & 1.1293 & 1.5403 \\
G.E.2 & 2.9493 & 4.9355 & 11.0193 \\
G.E.3 & 3.5447 & 4.0674 & 8.8883 \\
Pr. Ind. & 3.3321 & 3.9873 & 8.6826 \\
\hline
\end{tabular}

Fuente: Elaboración propia.

Tabla 6

Prueba de Kruskal-Wallis para los indicadores de riesgo

\begin{tabular}{cccc}
\hline Test & oMARBEN & oRENTECO & oRENTFIN \\
\hline Chi-cuadrado & 2.816 & 4.076 & 6.414 \\
gl & 2 & 2 & 2 \\
Sig. asintótica & 0.245 & 0.130 & 0.040 \\
\hline
\end{tabular}

Fuente: Elaboración propia. 
El ajuste de los resultados por el riesgo que soportan las empresas de cada grupo lo mediremos de forma análoga al procedimiento seguido por Cool y Schendel (1987) por medio del cociente entre el resultado medio y la desviación estándar del resultado obtenido a lo largo de los años que integran el periodo. De esta forma, la fórmula que tomaremos como referencia será:

$$
\frac{\mathrm{Xri1}}{\sigma \mathrm{ri1}}=\ldots \frac{\mathrm{Xrj2}}{\sigma \mathrm{rj2}}=\ldots \ldots \ldots \frac{\mathrm{Xrk3}}{\sigma \mathrm{rk3}}
$$

De igual forma que con los indicadores de resultados y de riesgo soportado, se presentan en las siguientes tablas los promedios de los resultados ajustados por el riesgo para las empresas que conforman los grupos y los promedios a nivel de la industria, así como la prueba de Kruskal-Wallis para los mismos indica- dores de resultados ajustados (Tabla 7 y Tabla 8)

En base a los resultados obtenidos en los test de Kruskal-Wallis para las dimensiones de resultados ajustados al riesgo de forma individual, donde el nivel crítico resulta significativo para la rentabilidad económica ajustada al riesgo, y para la rentabilidad financiera ajustada por el riesgo (menor a 0.05), deducimos que en cuanto a estos indicadores existen diferencias importantes, lo que nos lleva a aceptar parcialmente nuestra hipótesis. Respecto al margen de beneficios ajustado no existen indicios para aceptar la hipótesis de diferenciación en base a este indicador. Por lo cual, nos parece apropiado acometer algún tipo de comparación múltiple a posteriori para poder establecer en qué pares de grupos existen diferencias significativas. Entre las múltiples opciones disponibles, se ha

Tabla 7

Medias de los resultados ajustados por el riesgo

\begin{tabular}{cccc}
\hline Grupos & MARBEN ajustado & RENTECO ajustado & RENTFIN ajustado \\
\hline G.E.1 & 6.92 & 13.74 & 12.04 \\
G.E.2 & 2.34 & 2.00 & 2.29 \\
G.E.3 & 2.99 & 2.84 & 3.24 \\
Pr. Ind. & 3.16 & 3.45 & 3.70 \\
\hline
\end{tabular}

Fuente: Elaboración propia.

Tabla 8

Prueba de Kruskal-Wallis, resultados ajustados por el riesgo

\begin{tabular}{cccc}
\hline Test & MARBEN ajustado & RENTECO ajustado & RENTFIN ajustado \\
\hline Chi-cuadrado & 4.258 & 5.516 & 6.655 \\
gl & 2 & 2 & 2 \\
Sig. asintótica & 0.119 & 0.043 & 0.036 \\
\hline
\end{tabular}

Fuente: Elaboración propia. 
seleccionado el test de Bonferroni ${ }^{2}$, el cual nos permitirá comparar cada uno de los grupos estratégicos con sus similares, uno a uno, de manera de poder constatar las posibles diferencias de resultados ajustados al riesgo, pero ahora de forma más selectiva (Tabla 9 ).

En primer lugar, analizaremos las comparaciones en cuanto al margen de beneficios ajustados al riesgo. En cada una de las comparaciones del grupo de las multinacionales españolas con los demás grupos resulta significativa a un $93 \%$ -Sig. 0.07- revelando la posición favorable de las empresas de este grupo con respecto a sus competidores -la diferencia de medias nos ayuda a visualizar mejor las discrepancias-. Las diferencias

\section{Tabla 9}

\section{Test de Bonferroni para los resultados ajustados por el riesgo}

\begin{tabular}{|c|c|c|c|c|c|}
\hline $\begin{array}{c}\text { Variable } \\
\text { dependiente }\end{array}$ & $\begin{array}{c}\text { (I) Grupo a } \\
\text { comparar }\end{array}$ & $\begin{array}{c}\text { (J) Grupos } \\
\text { comparados }\end{array}$ & $\begin{array}{c}\text { Diferencia de } \\
\text { medias }(I-J)\end{array}$ & Error típico & Sig. \\
\hline \multirow{6}{*}{$\begin{array}{l}\text { MARBEN } \\
\text { ajustado al } \\
\text { riesgo }\end{array}$} & \multirow[t]{2}{*}{ G.E.1 } & G.E.2 & 4.5766 & 1.9758 & 0.076 \\
\hline & & G.E.3 & 3.9288 & 1.6791 & 0.072 \\
\hline & \multirow[t]{2}{*}{ G.E.2 } & G.E.1 & -4.5766 & 1.9758 & 0.076 \\
\hline & & G.E.3 & -.6477 & 1.2321 & 1.000 \\
\hline & \multirow[t]{2}{*}{ G.E.3 } & G.E.1 & -3.9288 & 1.6791 & 0.072 \\
\hline & & G.E.2 & .6477 & 1.2321 & 1.000 \\
\hline \multirow{6}{*}{$\begin{array}{l}\text { RENTECO } \\
\text { ajustado al } \\
\text { riesgo }\end{array}$} & \multirow[t]{2}{*}{ G.E.1 } & G.E.2 & 11.7450 & 2.4299 & 0.000 \\
\hline & & G.E.3 & 10.9052 & 2.0650 & 0.000 \\
\hline & \multirow[t]{2}{*}{ G.E.2 } & G.E.1 & -11.7450 & 2.4299 & 0.000 \\
\hline & & G.E.3 & -.8397 & 1.5153 & 1.000 \\
\hline & \multirow[t]{2}{*}{ G.E.3 } & G.E.1 & -10.9052 & 2.0650 & 0.000 \\
\hline & & G.E.2 & .8397 & 1.5153 & 1.000 \\
\hline \multirow{6}{*}{$\begin{array}{l}\text { RENTFIN } \\
\text { ajustado al } \\
\text { riesgo }\end{array}$} & \multirow[t]{2}{*}{ G.E.1 } & G.E.2 & 9.7466 & 2.1492 & 0.000 \\
\hline & & G.E.3 & 8.7966 & 1.8265 & 0.000 \\
\hline & \multirow{2}{*}{ G.E.2 } & G.E.1 & -9.7466 & 2.1492 & 0000 \\
\hline & & G.E.3 & -.9500 & 1.3403 & 1.000 \\
\hline & \multirow[t]{2}{*}{ G.E.3 } & G.E.1 & -8.7966 & 1.8265 & 0.000 \\
\hline & & G.E.2 & .9500 & 1.3403 & 1.000 \\
\hline
\end{tabular}

Fuente. Elaboración propia.

2 Como expresa Uriel (1995) existen diferentes procedimientos para realizar comparaciones múltiples de medias de poblaciones. Los métodos más conocidos para la construcción de intervalos de confianza conjuntos son los de Turkey, Scheffé y Bonferroni. Algunos autores proponen utilizar la aproximación de Bonferroni cuando el número de comparaciones no es muy elevado. 
parecen ser sensiblemente mayores con el grupo de las multinacionales extranjeras que con el grupo de las empresas especializadas En cuanto a las comparaciones entre los grupos multinacionales extranjeras y empresas especializadas no se pueden constatar diferencias significativas entre ellos en cuanto a esta dimensión de resultados ajustados al riesgo.

Seguidamente observamos las comparaciones de la variable rentabilidad económica ajustada por el riesgo, para cada uno de los pares de grupos. Se obtuvo una elevada significación $-0.00-$ al comparar el grupo multinacionales españolas con los demás grupos, reforzando los resultados obtenidos en la primera comparación. De igual forma, el G.E.1 parece ser más diferente del G.E.2 que del G.E.3, aunque por muy poco.

En el indicador rentabilidad financiera ajustada nuevamente las comparaciones entre el G.E.1 y los demás grupos resultan significativas -0.00 -, lo que indica que el G.E.1 es diferente a los demás. De nuevo las diferencias entre los grupos 1 y 2 son mayores que entre los grupos 1 y 3 .

Una vez comparadas las tres dimensiones, nos atrevemos a señalar que los resultados obtenidos por las empresas del G.E.1, sopesados los riesgos asumidos, son lo suficientemente elevados y diferentes a los de los demás grupos como para afirmar que es el grupo de empresas que presenta mayor estabilidad en cuanto a sus inversiones. En los indicadores rentabilidad económica ajustada al riesgo y rentabilidad financiera ajustada al riesgo, se han constatado mayores diferencias entre el G.E.1 y los demás grupos que con el indicador margen de beneficios ajustados al riesgo.
En base a estos resultados podemos señalar que las empresas del G.E.1 obtienen un mejor resultado que sus competidores, una vez sopesados los riesgos. Es probable que esta posición privilegiada la obtengan las empresas del G.E.1 apoyadas en una estrategia de innovación intermedia, en la cual, no asuman riesgos excesivos como en el caso de las empresas del G.E.2 que apuestan única y exclusivamente por la innovación radical, o como las del G.E. 3 conformado por laboratorios pequeños y medianos cuyas amenazas provenientes de las empresas grandes se magnifican al presentar una posición de desventaja en cuanto a recursos económicos. Sin embargo, el análisis de estos resultados no sólo debe ceñirse a la actividad innovadora de los grupos, sino también deben considerarse las otras estrategias adoptadas por las empresas. Las empresas del G.E.1 resultan ser las más diversificadas, lo que quiere decir que su riesgo asumido se encuentra más repartido entre diversos segmentos de la terapéutica. Esta situación también nos indica que es probable que estas empresas hagan un mejor uso de su conocimiento del mercado y aprovechen de mejor manera su red de distribución y ventas al tratar de abarcar más áreas.

\section{Conclusiones}

En esta investigación hemos obtenido un conjunto de grupos estratégicos sobre la base de aspectos vinculados con el tamaño de las empresas, su nivel de diversificación e internacionalización y la intensidad de la innovación, no obteniéndose diferencias significativas que pue- 
dan constatar la relación existente entre las estrategias empleadas por las empresas pertenecientes a los diferentes grupos estratégicos y los resultados económicos obtenidos por las mismas. Como aportación principal de la investigación podriamos destacar el hecho de haberse centrado en la industria farmacéutica, poco considerada en la literatura sobre grupos estratégicos, utilizándose además variables específicas de esta industria para establecer estos grupos.

En cuanto a los riesgos asumidos por las empresas de los grupos, hemos visto como a pesar de que las empresas del grupo de las multinacionales españolas asumen menores riesgos que los demás grupos, reflejado esto en una menor dispersión de los resultados, no hemos podido contrastar nuestra segunda hipótesis ya que estadísticamente no existen indicios para afirmarlo en dos de los tres indicadores de resultados.

Al sopesar los riesgos asumidos por cada empresa constatamos que existen diferencias en cuanto a los resultados ajustados por el riesgo y los grupos estratégicos. En tal sentido, las empresas multinacionales españolas obtienen mejores resultados una vez sopesados los riesgos en dos de los tres indicadores de resultados y con una significación del $93 \%$ para el indicador margen de beneficios ajustado. Estos resultados nos aclaran la importancia que representa el factor riesgo dentro de la industria farmacéutica al ver como el grupo de las multinacionales españolas, al encontrarse más diversificadas, al optar por una innovación de carácter incremental y al aprovechar su situación de "empresas sede" lo cual les permite minimizar algunos costes de transacción, obtienen unos resultados más estables que las empresas de los otros grupos.

En el caso de las "empresas especializadas", vemos como empresas de tamaño pequeño y mediano que no pueden apostar por una I+D radical, seguramente han encontrado alguna alternativa estratégica para alcanzar resultados similares al de sus competidores de los demás grupos estratégicos. Si bien es cierto que las multinacionales españolas y las multinacionales extranjeras obtienen mayores ingresos apoyadas en las economías de escala, no podemos afirmar que dichas empresas sean más eficientes que las demás al ser incapaces de diferenciarse en cuanto al porcentaje de beneficios obtenido.

En lo que respecta a la continuidad de la investigación, podría ser interesante tratar de establecer una clasificación de los grupos en base a variables internas de las empresas, es decir, en base a sus recursos y capacidades, para lo cual sería necesario determinar qué recursos internos se pueden constituir como fuente de ventaja competitiva dentro de esta industria. Finalmente, nos gustaría recalcar que los resultados obtenidos no deben considerarse de manera tajante debido a las innumerables peculiaridades que presenta la industria farmacéutica española producto de su alto nivel de intervención, y que la metodología de grupos estratégicos no sólo debe emplearse en el análisis de la relación grupo-desempeño empresarial, sino que también posee una elevada validez descriptiva, al ofrecer una visión de la heterogeneidad de los sectores industriales en función de determinadas variables estratégicas. 


\section{Referencias bibliográficas}

Bain, Joe (1956). Barriers to new competition. Estados Unidos: Harvard University Press.

Barney, Jay (1991). Firm resources and sustained competitive advantage. Journal of Management, $N^{\circ} 17, p p .99-$ 120.

Chi, Tailan (1994). Trading in strategic resources: Necessary conditions, transaction cost problems and choice of exchange structure. Strategic Management Journal, Vol. 15, pp. 271-290.

Claver, Enrique; Molina, José y Quer, Diego (2002). Grupos estratégicos, resultados empresariales y niveles de riesgo. Análisis empírico del sector de la construcción", Economía Industrial, $\mathrm{N}^{\circ} 345, \mathrm{pp} .147-158$.

Cool, Karely Dierickx, Ingemar (1993). Rivarly, strategic groups and firm profitability, Strategic Management Journal, Vol. 14, pp. 47-59.

Cool, Karel y Schendel, Dan (1987). Strategic groups formation and performance: the case of the U.S. pharmaceutical industry, 1963-1982, Management Science, Vol. 33, № 9, pp. 11021124.

Cool, Karel y Schendel, Dan (1988). Performance differences among strategic groups members, Strategic Management Journal, Vol. 9, pp. 207-223.

Dranove, David; Peteraf, Margaret y Shanley, Mark (1998). Do strategis groups exist? An economic framework for analysis, Strategic Management Journal, Vol. 19, pp. 1029-1044.

Farmaindustria (2003). Memoria anual de Farmaindustria y La industria farmacéutica en cifras, Farmaindustria, Madrid.

Fiegenbaum, Avi y Thomas, Howard (1990). Strategic groups and performance: The U.S. insurance industry 1970-84,
Strategic Management Journal, Vol. 11, pp. 197-215.

Flavian, Carlos y Polo, Yolanda (1999). Hacia la homogeneización de criterios en las investigaciones de grupos estratégicos, Cuadernos de Economía y Dirección de la Empresa, No 3, Enero-Junio, pp. 9-28.

Flavian, Carlos y Polo, Yolanda (2000). Perfiles de riesgo y resultados en la distribución comercial española, Economía Industrial, № 332, pp. 173-182.

Hair, Joseph; Anderson, Rolph; Tatham, Ronald y Black, William (1999). Análisis Multivariante. Quinta Edición. España: Prentice-Hall.

Hunt, Michael (1972). Competition in the major home appliance industry: 1960-1970. Tesis Doctoral, Harvard University.

Lewis, Pam y Thomas, Howard (1990). The linkage between strategy, strategic groups and performance in the U.K. retail grocery industry, Strategic Management Journal, Vol. 11, pp. 385-397.

McGee, John, Thomas, Howard y Pruet, Mark (1995). Strategic groups and the analysis of market structure and industry dinamics, British Journal of Management, $N^{\circ} 6$, pp. 257-270.

Pelayo, Yolanda y Vargas, Alfonso (2004). Grupos estratégicos en el sector envasador del aceite de oliva, Revista Europea de Dirección y Economía de la Empresa, Vol. 13, №3, pp. 93114.

Pérez, Rigoberto (1997). Análisis de datos económicos I. Métodos descriptivos. España: Pirámide.

Peteraf, Margaret (1993). The cornerstones of competitive advantage: a resourcebased view, Strategic Management Journal, Vol. 14, pp. 179-191.

Porter, Michael (1979). The structure within industries and companies' performance, The Review of Economics 
and Statistics, Vol. 6, No 1, pp. 214227.

Reger, Rhonda y Huff, Anne (1993). Strategic groups: a cognitive perspective, Strategic Management Journal, Vol. 14, pp. 103-124.

Shortell, Stephen y Zajac, Edward (1990). Perceptual and archival measures of miles and snow's strategic types: a comprehensive assessment of reliability and validity, Academy of Man- agement Journal, Vol. 33, pp. 817832.

Solá i Solá, Joaquim (2000). La industria farmacéutica españyola: estructura, estratégies i competitivitat, Document D’ Economía Industrial, Barcelona, España: Centre d'Economía Industrial.

Uriel, Ezequiel (1995). Análisis de datos. Series temporales y análisis multivariante. España: Editorial $A C$. 